\title{
Value at Risk Estimation Using Extreme Value Theory
}

\author{
Abhay K Singh, David E Allen and Robert J Powell \\ Edith Cowan University, Perth, Western Australia \\ Email: a.singh@ecu.edu.au
}

\begin{abstract}
A common assumption in quantitative financial risk modelling is the distributional assumption of normality in the asset's return series, which makes modelling easy but proves to be inefficient if the data exhibit extreme tails. When dealing with extreme financial events like the Global Financial Crisis of 2007-2008 while quantifying extreme market risk, Extreme Value Theory (EVT) proves to be a natural statistical modelling technique of interest. Extreme Value Theory provides well established statistical models for the computation of extreme risk measures like the Return Level, Value at Risk and Expected Shortfall. In this paper we apply Univariate Extreme Value Theory to model extreme market risk for the ASX-All Ordinaries (Australian) index and the S\&P-500 (USA) Index. We demonstrate that EVT can be successfully applied to Australian stock market return series for predicting next day VaR by using a GARCH $(1,1)$ based dynamic EVT approach. We also show with backtesting results that EVT based method outperforms $\operatorname{GARCH}(1,1)$ and RiskMetrics ${ }^{T M}$ based forecasts.
\end{abstract}

Keywords: Risk Modelling, Value at Risk, Extreme Value Theory, RiskMetrics ${ }^{T M}$, GARCH. 


\section{INTRODUCTION}

One of the major challenges in modelling VaR is the distributional assumption made for the return data series of the asset or portfolio, which is taken to be normal in most of the quantification approaches. The assumption of normality is not valid when the data series have heavy tails, which are characterised by extreme events left outside the bounds of a normal distribution when modelling VaR. The problem of the normality assumption of the return series, can be addressed by using the distribution free assumption of quantile modelling statistics, and tools such as quantile regression (Koenker and Bassett, 1978) or by applying extreme distribution based methods such as Extreme Value Theory (EVT).

With growing turbulence in the financial markets worldwide, evaluating the probability of extreme events like the GFC, has become an important issue in financial risk management. Quantification of the extreme losses in a financial market is important in current market conditions. EVT provides a comprehensive theoretical base on which statistical models describing extreme scenarios can be formed. The distinguishing feature of EVT is that it provides quantification of the stochastic behavior of a process at unusually large or small levels. Specifically, EVT usually requires estimation of the probability of events that are more extreme than any other that has been previously observed.

EVT, refers to the branch of statistics which deals with the extreme deviations from the mean of a probability distribution. EVT assesses the type of limiting probability distributions for the processes. In broad terms, EVT has two substantial ways of obtaining results or principal models: viz. the Block Maxima model (BMM) and Peak Over Threshold model (POT). Through the block maxima method, the asymptotic distribution of a series of maxima (minima) is modelled and the distribution of the standardized maximum is shown to follow extreme value distributions of Gumbel, Fréchet or Weibull distributions. The Generalized Extreme Value distribution (GEV) is a standard form of these three distributions, and hence the series is shown to converge to GEV. To analyse extreme market events, we are not always interested in maxima or minima of observations, but also in the behaviour of a large exceedance over a given threshold. The Peak over threshold method models a distribution of excess over a given threshold. EVT shows that the limiting distribution of exceedance is a Generalized Pareto Distribution or GPD (Coles, 2001;Coles and Tawn, 1991;1994, Franke, Härdle and Hafner, 2008 and Gilli and Këllezi, 2006). We will confine our focus to the POT method in this paper.

EVT is a well known technique in many fields of applied sciences including engineering and insurance (McNeil, 1999; Embrechts et al., 1999; Reiss and Thomas, 1997 and Giesecke \& Goldberg, 2005). Numerous research studies surfaced recently which analyse the extremes in the financial markets due to currency crises, stock market turmoils and credit defaults. The behaviour of financial series tail distributions has, among others, been discussed in Onour (2010), Gilli and Këllezi (2006), Loretan and Phillips (1994), Longin (1996), Daniels-son and de Vries (2000), Kuan and Webber (1998), Straetmans (1998), McNeil (1999), Jondeau and Rockinger (1999), Neftci (2000) and McNeil and Frey (2000). Diebold et al. (1998) discuss the potential of EVT in risk management.

Despite the promise of useful implementation of EVT in financial market analysis, it has only recently gained the attention of researchers in Australia. Chan and Gray (2006), Thomas et al. (2009) and Jeyasreedharan et al. (2009) are amongst the few studies to have used the technique. The lack of implementation of EVT methods on Australian markets act as our motivation to test it further on Australian market. This particular research paper also targets the United States market as natural comparators.

In this paper we model VaR in a dynamic two stage extreme value process with a GARCH $(1,1)$ model (McNeil and Frey, 2000), to forecast daily VaR with historical data in a moving window.

The rest of the paper is designed as follows; in section- 2 we give more details about EVT and the associated risk measures, in section-3 we outline the dynamic-EVT method for VaR and ES estimation. In Section-4 we provide a data description together with our research design and methodology. We discuss the results in section-5 and conclude in section- 6 .

\section{Extreme Value Theory and Extreme Risk Modelling}

EVT provides simple parametric models to capture the extreme tails of a distribution and to forecast risk. Mainly there are two broad methods of applying EVT: the first of which is based on the extreme value 
distributions of the Gumbel, Fréchet or Weibull distributions which are generalized as the Generalized extreme value distribution (GEV) and known as the Block Maxima (Minima) (BMM) approach, whilst the second is based on the Generalized Pareto Distribution (GPD) and is known as the peak over threshold (POT) approach. POT is considered more efficient in modelling limited data (Gilli and Këllezi, 2006; McNeil, Frey and Embrecht, 2005) as it fits the exceedances over a given threshold in a data set to GPD and hence is not as dependent on the requirement for large data sets as BMM. Our discussion of POT in this paper is adopted from Embrechts, Klüppelberg \& Mikosch (1997), Coles (2001), McNeil and Frey (2000), Gilli and Këllezi (2006), McNeil, Frey \& Embrechts (2005), Franke, Härdle and Hafner (2008).

\subsection{Generalized Pareto Distribution \& Peak Over Threshold (POT)}

Theorem 1. (Pickands (1975), Balkema and de Haan (1974)). For a large class of underlying distributions $F$, the excess distribution function $F_{u}$ can be approximated by GPD for an increasing threshold $u$.

$$
F_{u}(y) \approx G_{\xi, \sigma}(y), \quad u \rightarrow \infty
$$

$G_{\xi, \sigma}$ in theorem-1 is the Generalized Pareto Distribution (GPD) which is given by

$$
G_{\xi, \sigma}(y)= \begin{cases}\left(1+\frac{\xi}{\sigma} y\right)^{-1 / \xi} & \text { if } \xi \neq 0 \\ 1-e^{-y / \sigma} & \text { if } \xi=0\end{cases}
$$

for $y \in\left[0,\left(x_{F}-u\right)\right]$ if $\xi \geq 0$ and $y \in\left[0,-\frac{\sigma}{\xi}\right]$ if $\xi<0$. Here $\xi$ is the shape parameter and $\sigma$ is the scale parameter for GPD.

Definition 2. (Excess Distribution). For a random variable $X$ with $\mathrm{df} F$, the excess distribution over a threshold $u$ is given by

$$
F_{u}(y)=P(X-u \leq y \mid X>u)=\frac{F(y+u)-F(u)}{1-F(u)}=\frac{F(x)-F(u)}{1-F(u)},
$$

for $0<y<x_{F}-u$ where $x_{F} \leq \infty$ is the right endpoint of $F$ and $y=x-u$. $F_{u}$ is the conditional excess distribution function.

VaR and Expected Shortfall. If there is an extreme distribution $F$ with right endpoint $x_{F}$, we can assume that for some threshold $u, F_{u}(x)=G_{\xi, \sigma}(x)$ for $0 \leq x<x_{F}-u$ and $\xi \in \mathbb{R}$ and $\sigma>0$. For $x \geq u$,

$$
\begin{aligned}
\bar{F}(x) & =P(X>u) P(X>x \mid X>u) \\
& =\bar{F}(u) P(X-u>x-u \mid X>u) \\
& =\bar{F}(u) \bar{F}_{u}(x-u) \\
& =\bar{F}(u)\left(1+\xi \frac{x-u}{\sigma}\right)^{-1 / \xi}
\end{aligned}
$$

given $F(u)$, this gives a formula for tail probabilities. The inverse of (2.3) gives the high quantile of the distribution or VaR. For $\alpha \geq F(u)$, VaR is given by

$$
\operatorname{VaR}_{\alpha}=q_{\alpha}(F)=u+\frac{\sigma}{\xi}\left(\left(\frac{1-\alpha}{\bar{F}(u)}\right)^{-\xi}-1\right)
$$

For $\xi<1$ the ES is given by

$$
E S_{\alpha}=\frac{1}{1-\alpha} \int_{\alpha}^{1} q_{x}(F) d x=\frac{V a R_{\alpha}}{1-\xi}+\frac{\sigma-\xi u}{1-\xi}
$$

Analytical expressions for VaR and ES can also be defined as a function of estimated GPD parameters. Using (2.2) 


$$
F(x)=(1-F(u)) F_{u}(y)+F(u),
$$

if $n$ is the total observations and $N_{u}$ the number of observations above $u$ and we replace $F_{u}$ by the GPD and $F(u)$ by $\left(n-N_{u}\right) / n$, we get an estimator for tail probabilities (Smith, 1987)

$$
\hat{F}(x)=1-\frac{N_{u}}{n}\left(1+\frac{\hat{\xi}}{\hat{\sigma}}(x-u)\right)^{-1 / \hat{\xi}} .
$$

The inverse of (2.6) with a probability $p$ gives the VaR

$$
\widehat{V a R}_{p}=u+\frac{\hat{\sigma}}{\hat{\xi}}\left(\left(\frac{n}{N_{u}} p\right)^{-\hat{\xi}}-1\right)
$$

Using (2.5) the ES is given by

$$
\widehat{E S}_{p}=\frac{\widehat{V a R}_{p}}{1-\hat{\xi}}+\frac{\hat{\sigma}-\hat{\xi} u}{1-\hat{\xi}}
$$

In POT method GPD is fitted to the excess distribution (value above threshold a $u$ ) by MLE and the confidence interval estimates are calculated by profile likelihood and then the unconditional or static estimates for VaR and ES are calculated.

\section{EVT VAR AND ES-A DYNamic APPROACH}

McNeil and Frey (2000), proposed a dynamic VaR forecasting method based using EVT, their method makes use of GARCH modelling to model the current market volatility background which is further fed into VaR estimates obtained from the POT model fitted to residuals of a GARCH model. By use of GARCH models to forecast the estimates of conditional volatility the model provides dynamic one day ahead forecasts for VaR and ES for the financial time series.

Let $R_{t}$ the return at time $t$ be defined by the following stochastic volatility (SV) model

$$
R_{t}=\mu_{t}+\sigma_{t} Z_{t},
$$

where $\mu_{t}$ is the expected return on day $t$ and $\sigma_{t}$ is the volatility and $Z_{t}$ gives the noise variable with a distribution $F_{Z}(z)$ (commonly assumed to be standard normal). We assume that $R_{t}$ is a stationary process.

The most widely used suitable models are drawn from the ARCH/GARCH family. An autoregressive $\operatorname{GARCH}(1,1)$ process is given by

$$
\sigma_{t}^{2}=\alpha_{0}+\alpha_{1} \varepsilon_{t-1}^{2}+\beta \sigma_{t-1}^{2}
$$

where $\varepsilon=R_{t-1}-\mu_{t-1}, \mu_{t}=\lambda R_{t-1}, \alpha_{0}, \alpha_{1}, \beta>0, \beta+\alpha_{1}<1$ and $|\lambda|<1$.

In contrast to static risk modelling using EVT, where we model the unconditional distribution $F_{X}(x)$ and are interested in loss for $k$ days in general, the dynamic approach models the conditional return distribution conditioned on the historical data to forecast the loss over the next $k \geq 1$ days. If we follow the GARCH(1,1) model the one day ahead forecast of VaR and ES are calculated as:

$$
\begin{aligned}
\operatorname{VaR}_{q} & =\mu_{t+1}+\sigma_{t+1} \operatorname{VaR}\left(Z_{q}\right) \\
E S_{q} & =\mu_{t+1}+\sigma_{t+1} \operatorname{ES}\left(Z_{q}\right)
\end{aligned}
$$

With the assumption that $F_{Z}(z)$ is a known standard distribution, typically a normal distribution $Z_{q}$ can be easily calculated. The EVT approach (McNeil and Frey, 2000), instead of assuming $F_{Z}(z)$ to be normal applies the POT estimation procedure to this distribution of residuals.

For a return series at the close of day $t$ with time window of last $n$ returns $\left(R_{t-n+1}, \ldots, R_{t}\right)$ the method is implemented in following two steps. 
1. A GARCH $(1,1)$ model is fitted to the historical data by pseudo maximum likelihood estimation (PML) also known as Quasi-maximum likelihood estimation. The GARCH $(1,1)$ model in this step gives the residuals for step-2 and also 1 day ahead predictions of $\mu_{t+1}$ and $\sigma_{t+1}$.

2. EVT (POT method) is applied to the residuals extracted from step-1 for a constant choice of threshold $u$ to estimate $\operatorname{VaR}(\mathrm{Z})_{q}$ and $\mathrm{ES}(\mathrm{Z})_{q}$ to calculate the risk measures using equation-3.3.

The parameters of the GARCH model are estimated by the pseudo-maximum likelihood (PML) method. The likelihood of GARCH with a normality assumption is maximised to obtain parameter estimates $\hat{\theta}=\left(\hat{\lambda}, \hat{\alpha}_{0}, \hat{\alpha}_{1}, \hat{\beta}\right)^{T}$. Although this means fitting the model with a normality assumption, which is not always true for financial return data, PML usually generates fair estimates which are consistent and asymptotically normal (Gouriéroux, 1997). The POT method in step-2 is fitted using MLE.

\section{DAta \& Methodology}

We use a moving window of the last 1000 days log returns for ASX-All Ordinaries and S\&P-500 indices to forecast one day ahead $1 \%$ and $5 \%$ VaR estimates. The total data period is approximately 10 years (Jan-2000 to Dec-2010) containing 2850 daily log returns for both the indices, which gives us a total of 1850 predictions.

We chose a $90 \%$ quantile level as threshold, $u$ to fit the residuals from the GARCH(1,1) model to GPD. The forecasts from this method are compared with the forecasts from normal a GARCH $(1,1)$ where residuals are assumed to belong to normal distribution and to the RiskMetrics ${ }^{T M}$ model (J. P. Morgan, 1996).

We use a violation based backtesting method (McNeil and Frey, 2000) for the forecasted 1\% and 5\% VaR estimates. If we have a next day predicted quantile $\hat{r}_{q}^{t}$ and the actual return $r_{t+1}$, a violation is said to occur if $r_{t+1}>\hat{r}_{q}^{t}$, i.e. the actual loss is greater than the forecasted VaR. A binomial test for the success of these VaR forecasting models can be developed based on the number of violations. The test based on violations counts only two possible (binomial) outcomes of a violation or no violation. If $q$ is the quantile for VaR (95\% and $99 \%$ ) the estimated number of violations are given by $(1-q)$ Total Predictions (Trials). We will calculate a two sided binomial test of the null hypothesis against the alternative that the method has prediction errors and it underestimates (too many violations) or overestimates (too few violations) the conditional quantile.

\section{Results}

Figure-5.1 gives the plot of 1\% VaR estimates of ASX-All Ordinaries from the three models plotted with the actual return series for the prediction period. It is evident from the figure that the dynamic-EVT method closely follows the changing return dynamics of the market.

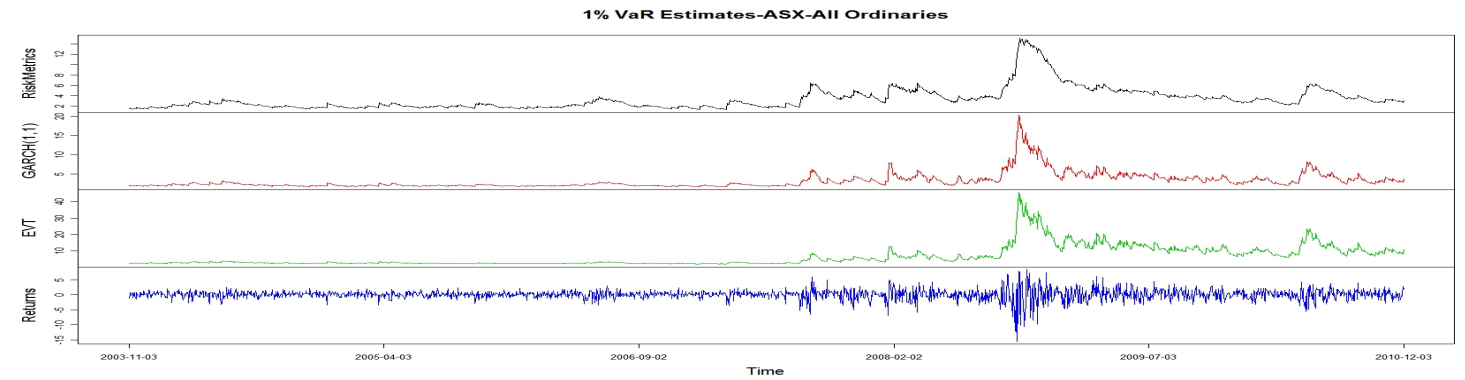

Figure 5.1: VaR Forecasts-ASX-All Ordinaries

Table-1 gives the backtest statistics for the models along with the two-sided p-value, a p-value greater 
than 0.05 shows the rejection of alternate hypothesis and hence is significant. The results show that apart from on one occasion (1\% VaR for S\&P-500) the dynamic-EVT method works better than all the other methods, in this case even when the p-value does not approve the method the method still has the least number of violations. Other significant result is that the other two models i.e. GARCH(1,1) and RiskMetrics $^{T M}$ fail for both quantile levels except RiskMetrics ${ }^{T M}$ for the ASX-All Ordinaries (q=0.95).

Table 1: Results-Backtesting VaR

\begin{tabular}{|c|c|c|}
\cline { 2 - 3 } \multicolumn{1}{c|}{} & ASX-All Ordinaries & S\&P-500 \\
\hline Total Predictions & 1850 & 1850 \\
\hline \multicolumn{3}{|c|}{$\mathrm{q}=0.99$} \\
\hline Expected & 18 & 18 \\
\hline Dynamic-EVT & $23(\mathbf{0 . 2 9})$ & $34(0.00)$ \\
\hline GARCH(1,1) & $42(0.00)$ & $49(0.00)$ \\
\hline RiskMetrics ${ }^{T M}$ & $43(0.00)$ & $45(0.00)$ \\
\hline \multicolumn{3}{|c|}{$\mathrm{q}=0.95$} \\
\hline Expected & 92 & 92 \\
\hline Dynamic-EVT & $81(\mathbf{0 . 2 4})$ & $104(\mathbf{0 . 2 2})$ \\
\hline GARCH $(1,1)$ & $123(0.00)$ & $115(0.02)$ \\
\hline RiskMetrics & \\
\hline & $107(\mathbf{0 . 1 2})$ & $117(0.01)$ \\
\hline
\end{tabular}

The forecasted period here includes the period of the GFC and it can be seen from the forecasted VaR that the method works well in the crisis period as well, which shows the capabilities of the EVT approach for modelling extreme market events. The dynamic model changes itself with changing market dynamics and hence the forecasted VaR values represent more closely the extreme risk of the market.

\section{Conclusion}

With the empirical analysis of this paper we demonstrated how we can use a GARCH based dynamicEVT approach to model VaR for short term forecasting. The dynamic-EVT method has the advantage of dynamically reacting to changing market conditions which is useful in getting better VaR forecasts. We show with our analysis that this method performs better than the other widely used methods of normal GARCH(1,1) and RiskMetrics ${ }^{T M}$, not only in normal market conditions but also in extreme market conditions such as the recent GFC.

\section{ACKNOWLEDGEMENT}

We are thankful to the Australian Research Council for funding support.

\section{REFERENCES}

Chan, K. F., \& Gray, P. (2009). Using Extreme Value Theory to Measure Value-at-Risk for Daily Electricity Spot Prices. International Journal of Forecasting, 22(2).

Coles, S. G. (2001). An introduction to Statistical Modelling of Extreme Values: Springer-Verlag.

Coles, S.G. \& Tawn, J.A. (1991), Modelling extreme multivariate events. J. R. Statist. Soc. B 53, $377-$ 392.

Coles, S.G. and Tawn, J.A. (1994), Statistical methods for multivariate extremes: An application to structural design (with discussion). Applied Statistics 43, 1-48.

Diebold, F. X., Schuermann, T., \& Stroughair, J. D. (1998). Pitfalls and opportunities in the use of extreme value theory in risk management. Journal of Risk Finance, 1, 30-35. 
Embrechts, P. (1999). Extreme value theory in finance and insurance. (Manuscript). Zurich: Department of Mathematics, ETH (Swiss Federal Technical University).

Embrechts, P., Klüppelberg, C. \& Mikosch, T. (1997), Modelling extremal events for insurance and finance, Springer, Berlin.

Engle, R. \& Nelson D. B. (1994). ARCH Models. In Robert F. Engle and Daniel McFadden (Eds.) Handbook of Econometrics (pp. 2959-3038).

Engle, R., \& Kroner, K. F. (1995). Multivariate simultaneous generalized ARCH. Econometric Theory $11,122-150$.

Franke, J., Härdle, K. W., \& Hafner, C. M. (2008) . Statistics of Financial Market: An Introduction (II ed.): Springer-Verlag Berlin Heidelberg.

Gilli, M., \& Këllezi, E. (2006). An Application of Extreme Value Theory for Measuring Financial Risk. Computational Economics, 27(2), 207-228.

Giesecke, K. \& Goldberg, L. R. (2005). Forecasting Extreme Financial Risk. In M. Ong (Ed.), Risk Management: A Modern Perspective: Elsevier Academic Publishing.

Gouriéroux, C. (1997). ARCH Models and Financial Applications. New York: Springer.

Jeyasreedharan, N., Alles, L. \& Yatawara, N. (2009). The Asymptotics of Extreme Returns in the Australian Stock Market. SSRN eLibrary.

Jondeau, E., \& Rockinger, M. (1999). The tail behavior of stock returns: Emerging versus mature markets. Documents de Travail 66: Banque de France.

Koenker, R. W., \& Bassett, G. Jr. (1978). Regression Quantiles. Econometrica 46(1), 33-50.

Kuan, C. H. \& Webber, N. (1998). Valuing Interest Rate Derivatives Con- sistent with a Volatility Smile. (Working Paper) University of Warwick.

Longin, F. M. (1996). The Assymptotic Distribution of Extreme Stock Market Returns. Journal of Business, 69, 383-408.

Loretan, M., \& Phillips, P. (1994). Testing the covariance stationarity of heavy-tailed time series. Journal of Empirical Finance, 1(2), 211-248.

McNeil, A. J. (1999). Extreme Value Theory for Risk Managers Internal Modelling and CAD (Vol. II, pp. 93-113): RISK Books.

McNeil, A., \& Frey, R. (2000). Estimation of Tail Related Risk Measure for Heteroscedastic Financial Time Series: An Extreme Value Approach. Journal of Empirical Finance 7, 271-300.

McNeil, A. J., Frey, R. \& Embrechts, P. (2005) Quantitative Risk Management: Concepts, Techniques and Tools. Princeton University Press.

J. P. Morgan. (1996). Riskmetrics. J. P. Technical document.

Neftci, S. N. (2000). Value at risk calculations, extreme events, and tail estimation. Journal of Derivatives, 23-37.

Onour, I. A. (2010). Extreme Risk and Fat-Tails Distribution Model: Empirical Analysis. Journal of Money, Investment and Banking.

Reiss, R. D., \& Thomas, M. (1997). Statistical Analysis of Extreme Values with Applications to Insurance, Finance, Hydrology and Other Fields: Birkhäuser Verlag, Basel.

R Development Core Team, R: A language and environment for statistical computing. http://www.Rproject.org, $R$ Foundation for Statistical Computing, ISBN 3-900051- 00-3, Vienna, Austria, 2003.

Straetmans, S. (1998). Extreme financial returns and their comovements. Phd. Dissertation, Tinbergen Institute Research Series, Erasmus University Rotterdam.

Thomas, S., Ramiah, V., Mitchell, H., \& Heaney, R. (2009). Seasonal factors and outlier effects in rate of return on electricity spot prices in Australia's National Electricity Market. Applied Economics, 43(3), 355-369. doi: 10.1080/00036840802570421 